\title{
Tuning the Interfacial Stoichiometry of InP Core and InP/ZnSe Core/Shell
}

\section{Quantum Dots}

Nayon Park, ${ }^{1}$ Forrest W. Eagle, ${ }^{1}$ Asher J. DeLarme, ${ }^{1}$ Madison Monahan, ${ }^{1}$ Talia LoCurto, ${ }^{1}$ Ryan

Beck, ${ }^{1}$ Xiaosong Li, ${ }^{1}$ and Brandi M. Cossairt ${ }^{1 *}$

${ }^{1}$ Department of Chemistry, University of Washington, Seattle, Washington, USA 98195-1700

*Email: $\underline{\text { cossairt@uw.edu }}$

\begin{abstract}
.
We demonstrate fine-tuning of the atomic composition of InP/ZnSe QDs at the core/shell interface. Specifically, we control the stoichiometry of both anions (P, As, S, and Se) and cations (In, $\mathrm{Zn})$ at the InP/ZnSe core/shell interface and correlate these changes with the resultant steadystate and time-resolved optical properties of the nanocrystals. The use of reactive trimethylsilyl reagents results in surface-limited reactions that shift the nanocrystal stoichiometry to anion-rich and improve epitaxial growth of the shell layer. In general, anion deposition on the InP QD surface results in a redshift in the absorption, quenching of the excitonic photoluminescence, and a relative increase in the intensity of the broad trap-based photoluminescence, consistent with delocalization of the exciton wavefunction and relaxation of exciton confinement. Time-resolved photoluminescence data for the resulting InP/ZnSe QDs show an overall small change in the decay dynamics on the ns timescale, suggesting the relatively low photoluminescence quantum yields may be attributed to the creation of new thermally activated charge trap states. Cluster-model density functional theory calculations show that the presence of core/shell interface anions give rise to electronic defects contributing to the redshift in the absorption. These results highlight a general strategy to atomistically tune the interfacial stoichiometry of InP QDs using surface-
\end{abstract}


limited reaction chemistry allowing for precise correlations with electronic structure and photophysical processes.

Keywords: indium phosphide, nanocrystal stoichiometry, surface chemistry, interface, composition, core/shell, photoluminescence

\section{Introduction.}

Advances in the synthetic optimization of InP-based quantum dots (QDs) have led this material to a position of prominence for commercial display applications. ${ }^{1-6}$ Recent demonstrations of near-unity quantum yield and $>20 \%$ external quantum efficiency in electroluminescent devices have placed InP on par with cadmium-based quantum dot emitters. ${ }^{7,8}$ The chemical design principles that underlie these advances, however, remain poorly understood. The atomistic synthesis of colloidal quantum dots that are defect-free, both structurally and electronically, has long been a topic of fundamental research and remains so even today. Typical solution-based hot injection and heat-up synthesis methods generate quantum dots that are marred by surface defects that create electronic trap states within the semiconductor band gap. ${ }^{9-14}$ These mid-gap states introduce both radiative and non-radiative relaxation pathways that compete effectively with the desired band-edge recombination process. Consequently, the need to understand and modulate the surface chemistry that governs these photophysical properties has risen to paramount importance in the field.

The effects of surface passivation and ligand type on the photoluminescence and charge

transfer properties have been abundantly reported. ${ }^{15-23}$ Recent studies on InP-based core/shell quantum dots have reported a diverse set of factors affecting the photoluminescence properties of 
emissive InP QDs, including uniformity of the InP core, ${ }^{24,25}$ thickness and composition of zinc chalcogenide shell, ${ }^{24-26}$ electron and hole wavefunction overlap, ${ }^{24,25}$ surface defects (such as oxidized species) or dangling bonds, ${ }^{7,23}$ and stacking faults at the core/shell interface due to lattice mismatch. ${ }^{27,28}$ The achievement of $100 \%$ photoluminescence quantum yield (PL QY) has been attributed to well-confined electron and hole wavefunctions through engineering the inner shell layer, ${ }^{25,29}$ removal of oxidative defects by etching with HF prior to shelling, ${ }^{7}$ a well-passivated surface on a stoichiometric InP core, ${ }^{8}$ and removal of indium defects in the shell by an additional washing step. ${ }^{8,30}$ The high sensitivity of optical properties to the core stoichiometry and interface morphology has been highlighted in recent computational studies. ${ }^{31,32}$ These considerations are influenced by the composition and the chemical environment at the core/shell interface, highlighting the need for further investigation on the nature of surface trap states and how to deterministically treat the quantum dot core en route to highly emissive, defect-free core/shell QDs.

In this study, we have sought to modify the interfacial stoichiometry of emissive InP QDs using surface-limited reaction chemistry to correlate interfacial composition with the PL properties of the resulting core and core/shell materials. Reactive silyl reagents containing the desired anion (P, Se, S, or As) were added to In-rich InP QDs capped with carboxylate ligands to obtain substoichiometric to anion-rich cores. Optical spectroscopy of the resultant core QDs indicates delocalization of the exciton wavefunction and relaxation of exciton confinement. The efficacy of the reactive silyl reagents in shifting the stoichiometry of shelled QDs and their impact on improving the epitaxial growth to reduce ensemble heterogeneity were demonstrated. Timeresolved photoluminescence (TR PL) spectroscopy revealed that interfacial modulation of InP/ZnSe QDs results in similar decay dynamics on the ns timescale, suggesting that changes in PL QY can be attributed to new defect-related pathways related to hole trapping on the ps timescale 
promoting non-radiative recombination. Further insight into the effects of surface stoichiometry of InP/ZnSe core/shell QDs was gained from analogous shelling experiments starting with purified Zn-treated InP QDs, which exhibited no notable differences from starting with In-rich InP QDs in the final PL traces, suggesting the role of $\mathrm{Zn}$ in the QD core in the final heterostructures is relatively insignificant compared to the immediate interface the core surface forms with the shell layer. The dynamics in all of these systems were found to be remarkably similar, and low temperature PL data supports the prevalence of trap-mediated non-radiative recombination process. Density functional theory (DFT) results indicate that modification of the stoichiometric composition at the core/shell interface can lead to the appearance of shallow hole traps dependent on the identity of the anion. These results highlight the role of the atomic composition of the QD core/shell interface and motivates further exploration of complex material systems fabricated through atomistic reaction chemistry.

\section{Results and Discussion.}

Anion-rich InP core QDs. First, In-rich, carboxylate-capped InP core QDs were synthesized from atomically precise $\operatorname{In}_{37} \mathrm{P}_{20}\left(\mathrm{O}_{2} \mathrm{R}\right)_{51}\left(\mathrm{R}=\mathrm{C}_{18} \mathrm{H}_{33}\right.$; oleate) clusters and purified to make a stock solution with a known In concentration. These InP cores were dried and redissolved in trioctylamine. Trioctylamine has recently gained traction in the synthesis of highly emissive InP-based quantum dots for its high boiling point $\left(365-367^{\circ} \mathrm{C}\right)$ and ease of removal with alcoholbased anti-solvents. ${ }^{4,7}$ In this reaction, it serves a double duty of maintaining the colloidal stability of the quantum dots upon surface treatment while circumventing surface etching that is observed with primary amines (Figure S1). 
Reactive trimethylsilyl ( $\left.\mathrm{SiMe}_{3}, \mathrm{TMS}\right)$ reagents containing the desired pnictide $(\mathrm{P}, \mathrm{As})$ or chalcogenide (S, Se) anion was suspended in trioctylamine. Half an equivalent of anion relative to In was used to yield InP cores with a maximally modified surface stoichiometry (Table 1). The addition of $\mathrm{P}\left(\mathrm{SiMe}_{3}\right)_{3}$ yielded particles that were still In-rich, but had a shifted stoichiometry of 1:0.8 In to P ratio, a 33\% increase from the starting 1:0.6 ratio of InP QDs. The upper limit for In:P ratio for a P-rich InP core that originally has a $3.1 \mathrm{~nm}$ diameter can be extrapolated from QD modelling by Kulik et al., ${ }^{33}$ and was found to be approximately 1:1.4 (calculations shown in SI, Text S1). For TMS arsenide, sulfide, and selenide, the surface reaction resulted in nanocrystals that exhibited a decrease in $\mathrm{P}$ content relative to In, while the added anions were present at a ratio that is almost equivalent to In, and an overall cation to anion ratio of 1:1.3 to 1:1.4.

Table 1. ICP-OES results showing In:P:E $(E=A s, S, S e)$ ratio of as-synthesized and surface aniontreated InP QDs normalized to In.

\begin{tabular}{lcccccc} 
& In & P & As & S & Se & In:anion \\
\hline InP & 1 & 0.6 & & & & $1: 0.6$ \\
InP+P & 1 & 0.8 & & & & $1: 0.8$ \\
InP+As & 1 & 0.5 & 0.9 & & & $1: 1.4$ \\
InP+S & 1 & 0.4 & & 0.9 & & $1: 1.3$ \\
InP+Se & 1 & 0.4 & & & 0.9 & $1: 1.3$
\end{tabular}

Using ${ }^{1} \mathrm{H}$ NMR spectroscopy, the reaction at the InP surface was further probed (Figure 1a). As-synthesized InP QDs can most simply be described as a stoichiometric core and an In-rich surface, capped by long chain carboxylate as the native ligand that is available for surface-limited reaction with the TMS reagent (Scheme 1). A stock of InP QDs was further purified by gel- 
permeation chromatography (x2) to ensure the removal of any excess ligands. The presence of a broad peak $(\delta=5.6 \sim 6.0 \mathrm{ppm})$ indicating bound oleate was confirmed. This peak shifts to approximately 5.3-5.6 ppm in the presence of trioctylamine. The reaction of purified InP QDs with $\mathrm{P}\left(\mathrm{SiMe}_{3}\right)_{3}$ shows growth of TMS ester over the course of the reaction with a concurrent decrease of the phosphine precursor peak. While the reaction hardly proceeds at room temperature, after one hour at $150{ }^{\circ} \mathrm{C}$, the reaction is mostly complete. The reaction of surface carboxylates and TMS phosphine proceeds and halts before all of the phosphine precursor is consumed. The reaction yield of TMS ester is approximately $40 \%$, while $45 \%$ of the $\mathrm{P}\left(\mathrm{SiMe}_{3}\right)_{3}$ remains unreacted, which is in good agreement with the ICP-OES results. Additional evidence for the removal of the native carboxylate ligands is the evolution of a multimodal resonance that includes a sharp component in the alkene region as the ligand becomes unbound from the nanocrystal surface and forms TMS ester. Similarly, the reaction of purified InP QDs with $\mathrm{Se}\left(\mathrm{SiMe}_{3}\right)_{2}$ was monitored by ${ }^{1} \mathrm{H}$ NMR spectroscopy. The conversion yield of TMS selenide was calculated to be $65 \%$, which is significantly higher than that of $\mathrm{P}\left(\mathrm{SiMe}_{3}\right)_{3}$ and is consistent with the high ratio of Se incorporated in the surface-modified InP QDs from the ICP results (Table 1).

Scheme 1. Proposed surface reactions between carboxylate/carboxylic acid-capped InP QDs and $\mathrm{P}\left(\mathrm{SiMe}_{3}\right)_{3}$.
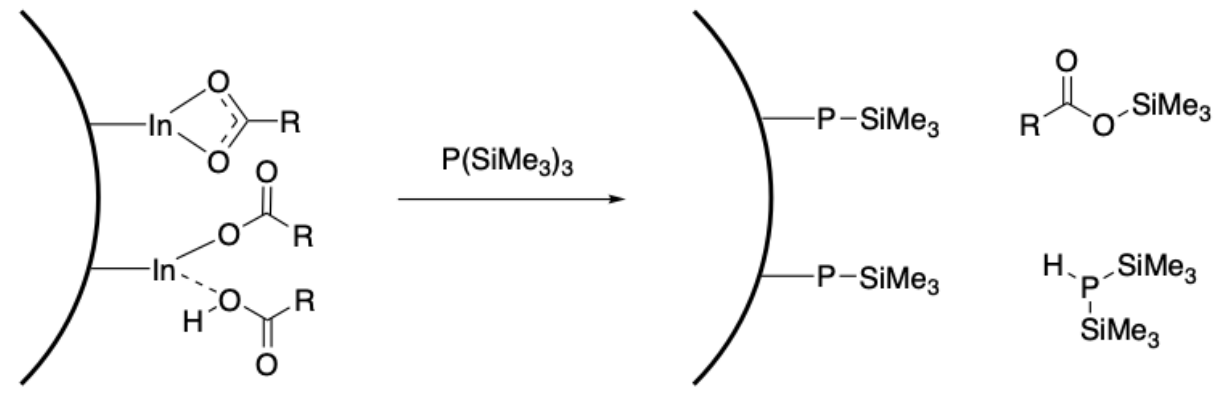
The observed optical properties from UV-vis and PL spectroscopy reveal a redshift by 17 nm with the addition of $\mathrm{P}\left(\mathrm{SiMe}_{3}\right)_{3}$ (Figure 1b), which can be attributed to the growth of the InP core as previously reported in the literature. ${ }^{8}$ The excitonic PL intensity was diminished without significantly perturbing the broad, redshifted trap PL. This characteristic trap PL feature of InP QDs has previously been ascribed to the emission from hole trap states arising from surface $\mathrm{P}$ dangling bonds. ${ }^{14}$ The addition of $\mathrm{As}\left(\mathrm{SiMe}_{3}\right)_{3}$ to the $\mathrm{InP}$ core resulted in a similar redshifted absorbance and quenching of excitonic PL while trap PL persisted, although the absorbance peak appeared qualitatively much less defined.
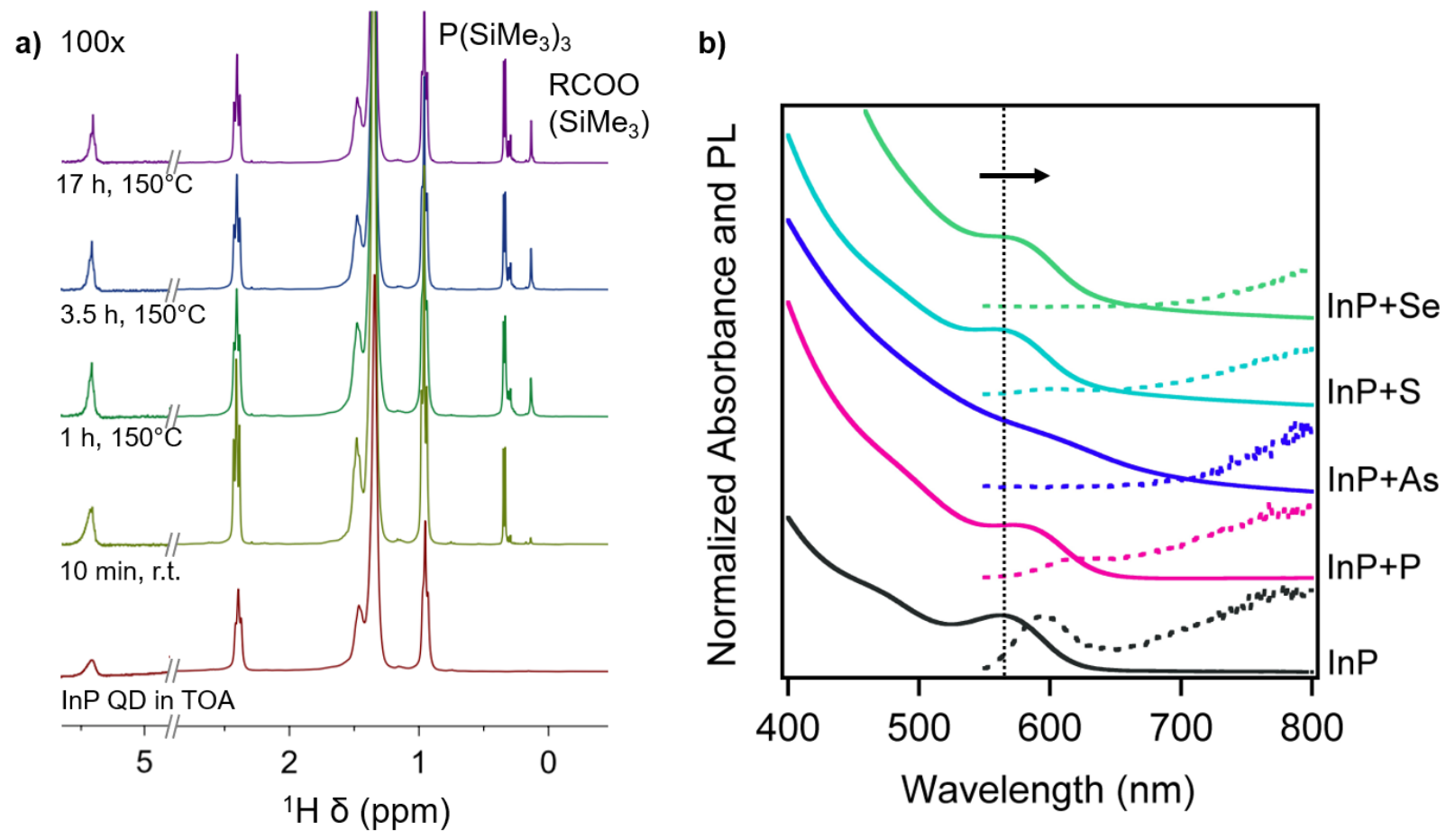

Figure 1. InP QD surface treatment with TMS anion reagents. a) ${ }^{1} \mathrm{H}$ NMR spectra showing the evolution of the reaction of carboxylate-capped InP QD with $\mathrm{P}\left(\mathrm{SiMe}_{3}\right)_{3}$ obtained $\left(300 \mathrm{MHz}, \mathrm{C}_{6} \mathrm{D}_{6}\right.$, $298 \mathrm{~K}$, delay time of 20s). The oleate peak centered around $5.5 \mathrm{ppm}$ was amplified by 100x for visibility. b) UV-Vis (solid) and PL (dotted) spectra of native (black), P (pink), As (navy), S (teal), 
and Se-treated (green) InP QDs. The vertical dotted line indicates the absorbance max of native InP QD to guide the eye for redshifted absorbance of anion-treated InP QDs.

The addition of bis(trimethylsilyl) sulfide (or selenide) afforded S- (or Se-) treated, anionrich InP cores. The formation of InP cores with these chalcogenides coating the surface is of interest as they are commonly used as a component of the wide band gap shell material, including $\mathrm{Zn}$ and $\mathrm{Cd}$ chalcogenides. Both S- and Se-treated InP cores exhibited a redshift in the absorbance, while the excitonic PL was significantly diminished (Figure 1b). The broad, redshifted trap PL persisted for both, remaining as a notable feature of these core QDs with anion-rich surfaces.

Structural characterization of the surface-modified InP cores by powder X-ray diffraction (XRD, Figure S2) confirms that these core QDs retain the zinc blende InP structure with no observable change in FWHM. It is worth noting the (111) plane of the S-treated core shifts to higher $2 \theta$ by $0.4^{\circ}$ suggestive of a contracted lattice due to the surface strain by the smaller $\mathrm{S}$ atoms. TEM (transmission electron microscopy) images corroborate no notable changes in the size of the nanocrystals from the original $3.1 \mathrm{~nm}$ diameter (Figure S3).

With the knowledge that the surface anion treatment did not change the size, morphology, or the crystal structure of the InP QDs to any significant degree, the observation of the redshift in the absorbance can be attributed to a change in electronic structure as a result of the shifted stoichiometry. More specifically, the redshift suggests delocalization of the charge carrier wavefunction resulting in relatively softer confinement of the exciton in the core. This is consistent with theoretical investigations by Califano et al., ${ }^{31}$ who concluded that P-rich InP QDs have delocalized electrons and localized holes, resulting in an overall decreased wavefunction overlap. Similar phenomena have been reported by Brutchey et al. where the observed redshifts with 
chalcogenol ligands installed on the surface of CdSe QDs were attributed to relaxed quantum confinement resulting from the coupling between the HOMO of the chalcogenol and the QD hole wavefunction. ${ }^{34}$ It was proposed that chalcogenol ligands attract the hole and localize hole density at the surface. Because such spatial separation would naturally result in reduction of exciton wavefunction overlap, the quenching of excitonic PL is expected, and we propose this as a reasonable explanation for the PL quenching we observe in the anion-treated InP cores.

Scheme 2. Shelling scheme of interface controlled InP/ZnSe QDs.

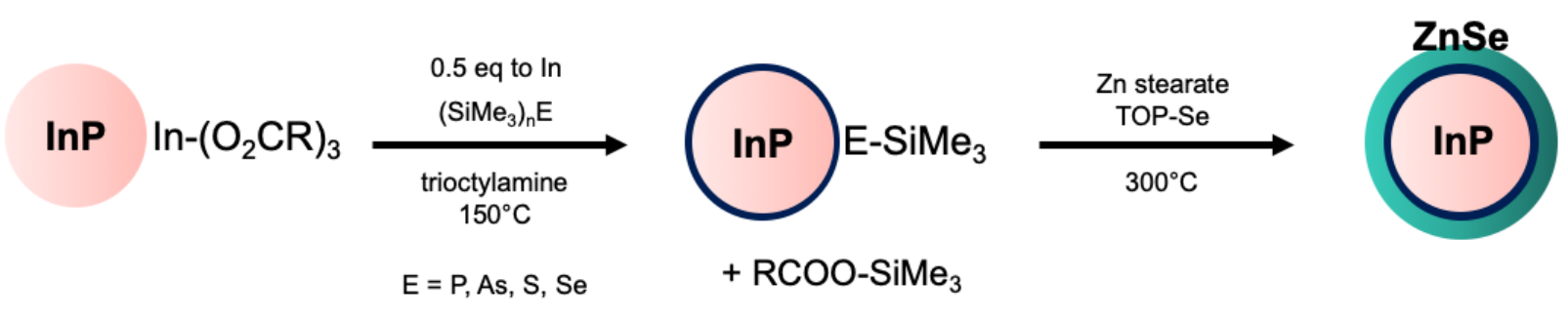

Anion-rich interface in core/shell QDs. In situ shelling of the anion-rich InP QDs was performed to investigate the effects of the modified interfaces on the luminescence and photophysical properties of the core/shell QDs. A wider band gap ZnSe shell for type-I band alignment (confinement of exciton to the core) with $\mathrm{InP}$ was chosen to standardize the shelling procedure, for structural simplicity, and a better lattice match with the InP core compared to $\mathrm{ZnS}$ (3.4\% vs. 7.7\%). Shelling was carried out (Scheme 2) by first taking purified InP QDs dissolved in trioctylamine and slowly injecting 0.5 eq. (vs. In) of the reactive silyl reagent containing P, As, $\mathrm{Se}$, or $\mathrm{S}$ at $150{ }^{\circ} \mathrm{C}$ for an hour. A $\mathrm{ZnSe}$ shell was grown on the surface-modified cores using temporally separated slow injection of $\mathrm{Zn}$ and Se precursors, each at $220{ }^{\circ} \mathrm{C}$ and $300{ }^{\circ} \mathrm{C}$, respectively. The excitonic PL was recovered for all samples upon the addition of $\mathrm{Zn}$ and continued to increase in intensity throughout the growth of the $\mathrm{ZnSe}$ shell. The observation of increased PL 
intensity upon the addition of $\mathrm{Zn}$ can be attributed to the passivation of the surface anion sites that serve as hole traps as has been seen previously in both II-VI and III-V systems. ${ }^{13,35}$

The resultant InP/ZnSe core/shell QDs exhibited varying PL efficiencies and a range of absorption and PL wavelengths. The highest PL QY of $41 \%$ straight out of synthesis was achieved by the control sample where no interfacial anion was added prior to shelling. The PL QY was improved to $75 \%$ upon initial purification, likely due to removal of a population of unshelled InP QDs. This PL QY was reduced to 15\% upon size-selective precipitation that narrowed the emission linewidth. Size-selective precipitation was necessary to proceed with further characterization of these samples to probe the impact of interfacial stoichiometry in a homogeneous population. The change in emission linewidth following size-selective precipitation was most pronounced in the control sample, suggesting heterogeneous shell growth that results in the broadening of the linewidth. The normalized PL spectra of all samples before and after size-selective precipitation are shown in Figure 2a. Notably, the PL linewidth and the line shape showed minimal change after size-selective precipitation for the As, S, and Se-rich interface core/shell QDs, suggesting a more controlled and uniform growth upon synthesis compared to the traditional shelling approach.

b) 

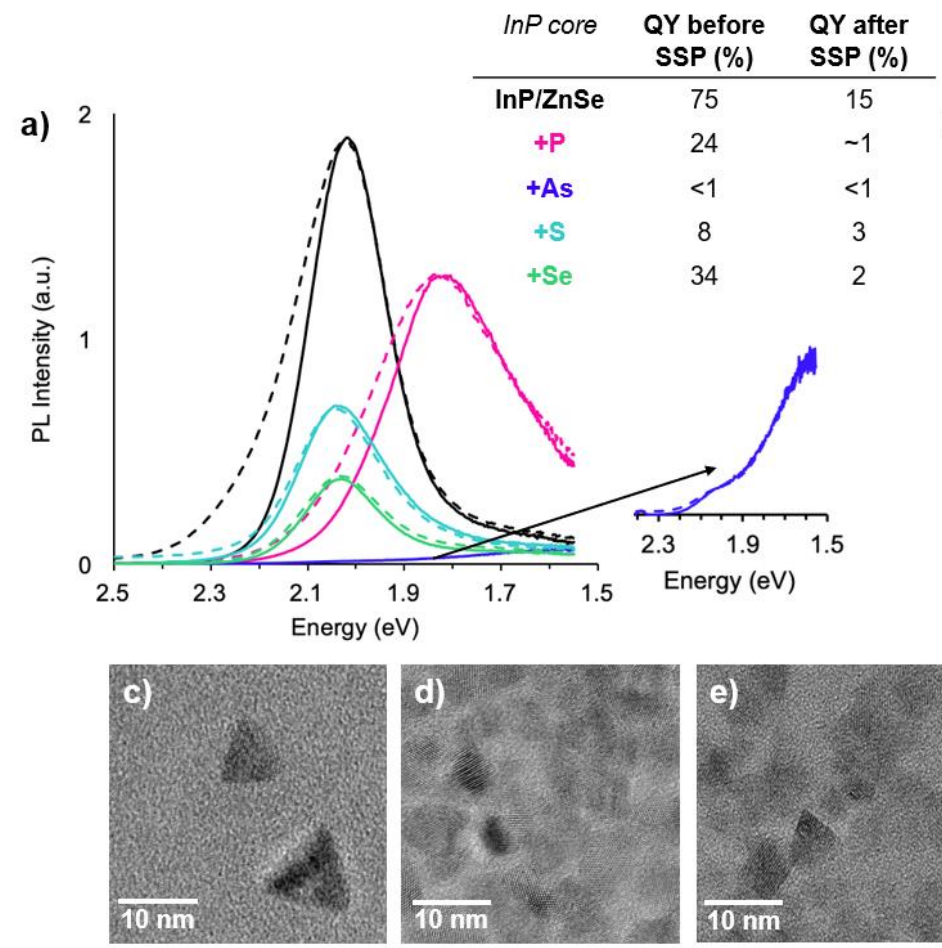

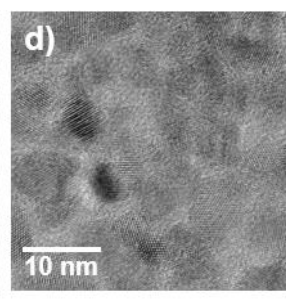

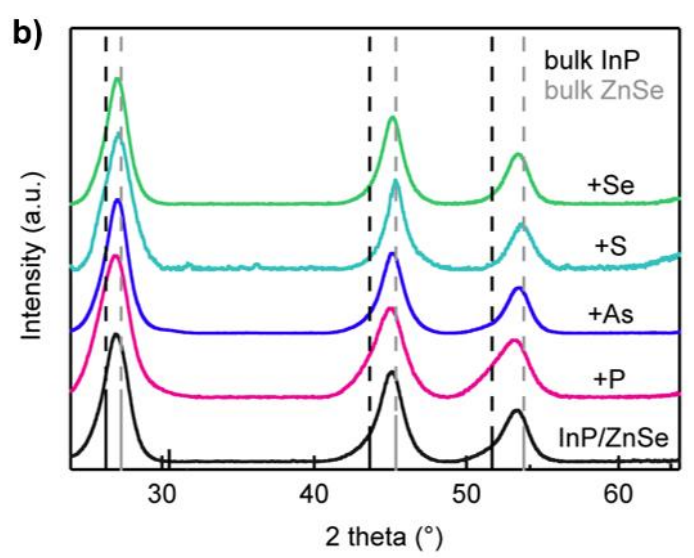
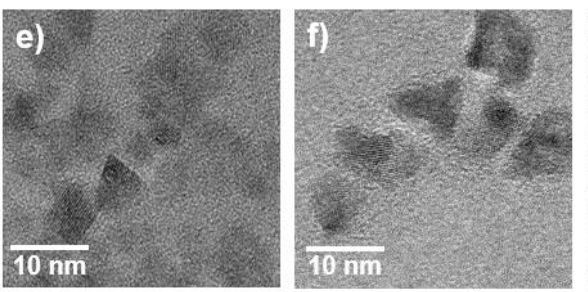

Figure 2. InP/ZnSe QDs with modulated interfacial anion composition. a) PL spectra of anionrich interface before size-selective precipitation (dotted, normalized to solid trace) and after (solid) size-selective precipitation. Quantum yields before and after size-selective precipitation are noted in the legend. InP+P/ZnSe sample quickly oxidized upon exposure to air precluding accurate measurement. b) Powder XRD patterns of InP/ZnSe QDs with anion-rich interface compared with traditional InP/ZnSe QDs exhibiting a shift from InP (PDF \#01-070-2513) toward ZnSe (PDF \#04001-6858), and c-g) TEM images of control, P, As, S, and Se-interface core/shell QDs.

Further insight was gained from elemental and structural analysis of the shelled particles purified by size-selective precipitation. Elemental analysis by ICP-OES indicated retention of the anions that were post-synthetically added to the purified InP QD surface (Table 2). The P-treated interface resulted in an In:P of 1:1. It is evident from the atomic ratios that the anions employed in 
core surface treatment were incorporated in the final core/shell structure, with the ratio of the shelling layer representative of a 1-2 monolayer shell. The expected core/shell structure is corroborated by powder XRD (Figure 2b), where the zinc blende InP/ZnSe QD structure is observed across all samples, with the InP reflections exhibiting shifts toward bulk ZnSe, indicating successful shelling and reliably homogeneous populations following size-selective precipitation. For the sample with a P-rich interface, the asymmetry of the peaks at $43.6^{\circ}(220)$ and $51.7^{\circ}(311)$ may be attributed to stacking disorder within the nanocrystal. ${ }^{36,37}$ We propose that inhomogeneous strain due to uneven compositional distribution or alloying can give rise to defect-related nonradiative recombination. TEM analysis shows nanocrystals of sizes between 7.0-7.5 $\mathrm{nm}$ in diameter and highly crystalline lattices despite their irregular morphology (Figure 2c-g).

Table 2. The atomic compositions of InP/ZnSe QDs with anion-modulated interfaces obtained from ICP-OES. The molar ratios are normalized to moles of In.

\begin{tabular}{lcccccc} 
& In & P & As & S & Se & Zn \\
\hline InP/ZnSe & 1.0 & 0.3 & & & 6.8 & 7.1 \\
InP+P/ZnSe & 1.0 & 1.0 & & & 5.2 & 5.8 \\
InP+As/ZnSe & 1.0 & 0.3 & 2.7 & & 5.6 & 5.0 \\
InP+S/ZnSe & 1.0 & 0.5 & & 1.0 & 3.8 & 4.6 \\
InP+Se/ZnSe & 1.0 & 0.4 & & & 9.1 & 8.6
\end{tabular}

These samples were further probed by time-resolved photoluminescence spectroscopy (TR PL) to study the effects of the modified interfaces on the charge carrier dynamics. The PL decay data were collected over a 200 ns window and were each fit with biexponential functions. Notably, the samples with S- and Se-rich interfaces showed a decreased lifetime in the faster component when compared to control InP/ZnSe QDs. The As-rich interface sample exhibited very similar 
decay dynamics to the control InP/ZnSe QDs, while the P-rich interface sample showed a relatively larger magnitude of the slower time component, increasing the weighted lifetime. The decreased lifetime of the faster component observed for the chalcogenide-rich interfaces may suggest the emergence of new processes such as trapping at chalcogen-derived defect states. Lower PL QYs for samples with modified interfaces support the creation of new pathways that facilitate nonradiative recombination. While steady-state and time-resolved PL offer insight that a shifted atomic composition at the core/shell interface, even with submonolayer coverage, can alter the optical and photophysical properties in the ns timescale, the differences in the weighted lifetimes are small overall and show no change in the longer timescale. PL quenching evident in the overall low QY of the samples suggests behavior of the charge carriers not reflected in the lifetime and leads us to speculate that the ensembles contain populations of QDs that are non-luminescent. ${ }^{38}$ In light of recent NMR studies of Cd-treated InP QDs ${ }^{39}$ where the presence of a mixture of surface species including surface phosphates was confirmed, it seems that this approach to surface modification on an already defective core further reinforces the defect-ridden interface, resulting in poor optical properties. Removal of oxidized surface defects with HF and subsequent addition of anions was attempted and resulted in similar final PL QYs (Figure S4), necessitating further exploration of the surface chemistry needed to form an ideal interface. 


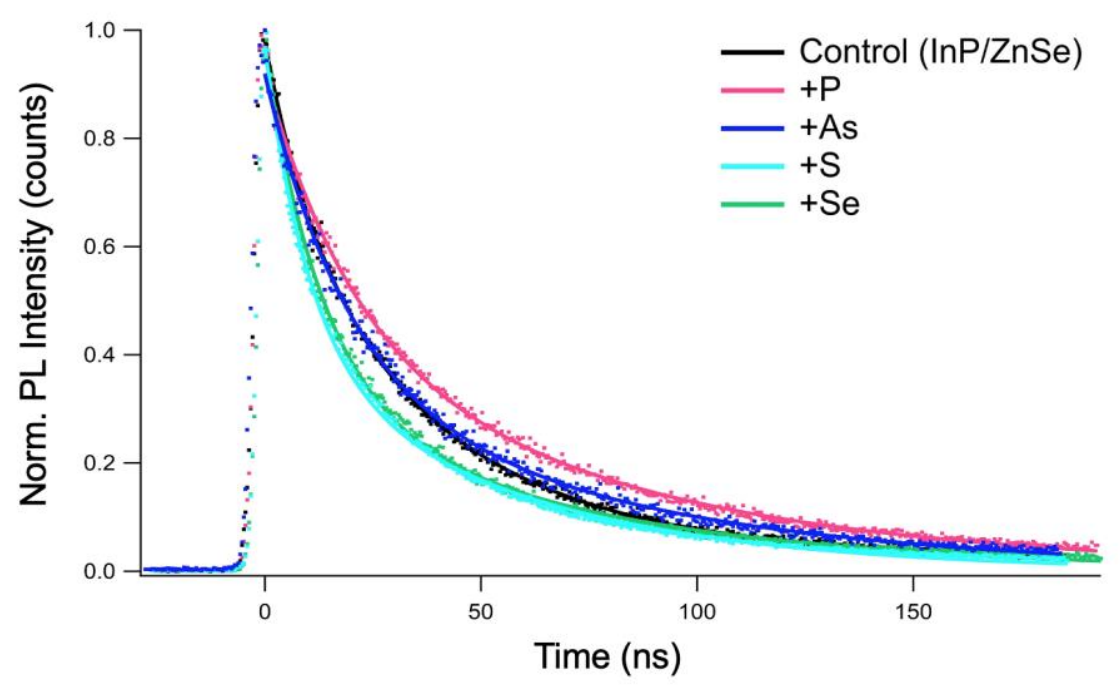

Figure 3. Excitonic PL decay dynamics of InP/ZnSe QDs with modulated interfaces measured at room temperature. Transient PL spectra were obtained by integrating streak camera data over 200 ns between 2.14-1.97 eV (black, navy, teal, green traces) or 1.91-1.77 eV (pink trace).

Table 3. Parameters fit to biexponential function for excitonic PL decay dynamics measured at room temperature.

\begin{tabular}{lccccc} 
Sample & $\mathbf{A}_{\mathbf{1}}$ & $\boldsymbol{\tau}_{\mathbf{1}}(\mathbf{n s})$ & $\mathbf{A}_{\mathbf{2}}$ & $\boldsymbol{\tau}_{\mathbf{2}}(\mathbf{n s})$ & $\boldsymbol{\tau}_{\text {weighted }}(\mathbf{n s})$ \\
\hline InP/ZnSe & 0.61 & 19 & 0.35 & 67 & 37 \\
InP+P/ZnSe & 0.48 & 20 & 0.44 & 78 & 48 \\
InP+As/ZnSe & 0.55 & 19 & 0.36 & 76 & 38 \\
InP+S/ZnSe & 0.56 & 10 & 0.41 & 55 & 28 \\
InP+Se/ZnSe & 0.63 & 13 & 0.33 & 68 & 32
\end{tabular}

To better understand the nature of the trap states and the charge carrier dynamics, we measured the PL spectra at low temperature $(77 \mathrm{~K})$ to compare to room temperature PL (Figure S5). Generally, it is evident that when the temperature is decreased, the excitonic PL blueshifts as expected from the Varshni relation. ${ }^{40}$ For the samples with modulated interface compositions, the overall PL intensities tend to increase. Qualitatively, the relative increase of trap PL is higher than 
that of excitonic PL. This suggests a thermally mediated de-trapping mechanism at room temperature, which has been previously reported with InP cores with implication for defects from dangling bonds either from In or P surface atoms. ${ }^{14}$ The anion-derived trap PL we observed in the surface modified InP cores is suppressed at room temperature upon shelling, but these electronic defects are evident in the low temperature spectra.

Tuning surface anion composition of optically bright, Zn-rich InP cores. We extended the sample set to include Zn-rich InP cores exhibiting 20\% PL QY as the starting material for interface tuning and shelling. $\mathrm{Zn}$-rich InP cores are best described as stoichiometric InP cores with $\mathrm{Zn}$ carboxylates as surface ligands. In this set of experiments, TMS phosphine and selenide were specifically chosen to modulate the interfacial stoichiometry. Additionally, as an analogue to $\mathrm{Se}\left(\mathrm{SiMe}_{3}\right)_{2}$, TOP-Se was used for comparison. Upon introduction of the anion precursor, the absorption peak was redshifted across all samples accompanied by an increase in the PL intensity. With each subsequent addition of the shelling precursors, the absorption and PL maxima continued to redshift. The final absorption and PL wavelengths for these interface modified InP/ZnSe QDs were strikingly similar between In-rich and Zn-rich InP cores (Figure S6a). P-treated InP-Zn core QDs displayed the largest redshift overall, while surface treatment with $\mathrm{Se}\left(\mathrm{SiMe}_{3}\right)_{2}$ or TOP-Se resulted in PL maxima similar to what is observed for $\mathrm{InP} / \mathrm{ZnSe}$ without surface anion treatment, with the overall PL profiles nearly overlapping. Starting with bright core QDs achieved with Zn treatment led to higher PL QYs in the final core/shell QDs overall and less loss of PL QY upon size-selective precipitation, suggesting that the emissive quality of the starting core material is critical to the final optical properties and photostability.

Elemental analysis by ICP-OES demonstrates that both P and Se introduced by the reactive silyl precursors were successfully incorporated into the final core/shell QDs (Table S1). In both 
cases, an overall anion-rich stoichiometry was obtained. Interestingly, there was a difference in the atomic ratios between the $\mathrm{Se}\left(\mathrm{SiMe}_{3}\right)_{2}$ and TOP-Se treated samples resulting in a reversed cation to anion ratio. This indicates that the reactive silyl precursor that targets direct reaction with surface carboxylates renders a different surface chemistry and atomic composition that persists after the shell growth. The expected zinc blende structure of the final core/shell QDs and the size and morphology of the particles were corroborated by the powder XRD patterns and TEM.

The excitonic PL decay dynamics of the InP/ZnSe QDs made from Zn-treated InP cores were investigated (Figure S6b, Table S2). Compared to the control InP/ZnSe sample, P-rich interface core/shell QDs showed a reduction in the amplitude of the short time component and a relative increase of the slower time component with an extended lifetime, similar to the decay dynamics when the In-rich InP core was shelled. The samples modified with Se precursors, however, exhibited very similar excitonic PL dynamics where the decay curve overlaps with that of the control InP/ZnSe sample. Here, the shift in stoichiometry of the cation to anion ratio induced by the different Se precursors evident in ICP-OES results does not have any observable changes in this timescale, suggesting the loss in PL QY can again be attributed to hole-associated trapping on a faster timescale and the creation of non-radiative decay pathways and/or dark fractions. This also suggests that the immediate interface formed with the InP core (i.e., with $\mathrm{Zn}$ carboxylate) is more influential to the photophysics of heterostructure QDs. The dynamics in all of these systems remain remarkably similar. The low temperature PL of these samples (Figure S5) shows a notably different trend from the samples made from In-rich InP cores. At lower temperature, the excitonic PL intensity decreased while the trap PL intensity increased. This is consistent with the observation of the InP/ZnSe control sample, supporting the importance of immediate interfacial structure (surface anion vs cation) at the core/shell interface. Interestingly, InP-Zn+P/ZnSe sample showed 
a redshift upon lowering the temperature, which to our knowledge has not previously been observed in shelled InP literature and is a surprising result given the Varshni relation. While less common, an increase in band gap with increasing temperature has been previously observed for $\mathrm{Pb}$ chalcogenide QDs and has been attributed to an unconventional trapped exciton state ${ }^{41}$ or exciton dark-bright state splitting. ${ }^{42}$

Cluster-models of core and core/shell InP QDs. In order to gain further insight into the electronic structure of the surface modified core and core/shell QDs, we turned to density functional theory. Calculations were conducted using the Gaussian software package $(\mathrm{G} 16 . \mathrm{B} 01)^{43}$ using the Perdew, Burke, and Ernzerhof hybrid functional (PBE0) ${ }^{44-46}$. Quasi-spherical InP QD, $\operatorname{In}_{77} \mathrm{P}_{77}$ (diameter $\sim 2 \mathrm{~nm}$ ) was constructed using the bulk zinc blende crystal structure. The pure InP structure conformed to a $\mathrm{C}_{3 v}$ symmetry before optimization. Core/shell structures were built from the InP core by exchanging surface In with $\mathrm{Zn}$ and $\mathrm{P}$ with Se to form $\operatorname{In}_{\mathrm{x}-\mathrm{y}} \mathrm{P}_{\mathrm{x}-\mathrm{y}} / \mathrm{Zn}_{\mathrm{y}} \mathrm{Se}_{\mathrm{y}}(\mathrm{d} \sim 2$ $\mathrm{nm}$ for $\left.\mathrm{In}_{10} \mathrm{P}_{10} / \mathrm{Zn}_{67} \mathrm{Se}_{67}\right)$. In order to generate the anion-rich models $\left(\operatorname{In}_{10} \mathrm{P}_{10-\mathrm{y}} \mathrm{E}_{\mathrm{y}} / \mathrm{Zn}_{67} \mathrm{Se}_{67}, \mathrm{E}=\mathrm{As}\right.$, $\mathrm{S}$, and Se), the previously optimized $\operatorname{In}_{10} \mathrm{P}_{10} / \mathrm{Zn}_{67} \mathrm{Se}_{67}$ core/shell model had the interfacial $\mathrm{P}$ atoms replaced. Surface dangling bonds were terminated using a pseudo-hydrogen capping scheme to compensate surface ions $( \pm 1 / 3$ for the non-shelled $\mathrm{InP}$ systems $)$ resulting in $(\mathrm{InP})_{77} \mathrm{H}_{108}$ structure for the non-shelled system. The calculated band gaps of the $(\operatorname{InP})_{77} \mathrm{H}_{108}$ and the $(\mathrm{InP})_{10} /(\mathrm{ZnSe})_{67} \mathrm{H}_{108}$ structures were $3.89 \mathrm{eV}$ and $3.77 \mathrm{eV}$, respectively.

Surface modified core structures were constructed by replacing surface P atoms with As atoms resulting in an $\operatorname{In}_{77} \mathrm{P}_{23} \mathrm{As}_{54}$ structure. The addition of the surface As atoms resulted in a decrease in the band gap (by $0.16 \mathrm{eV}$ ) in comparison to the $\operatorname{In}_{77} \mathrm{P}_{77}$ structure (Figure 4, Table S3). This resulted in a redshift in the TD-DFT absorption spectrum (by $0.13 \mathrm{eV}$ ), which is consistent with the experimental data. It is important to note that the models investigated are generally blue 
shifted in relation to the experimental systems as they are more quantum confined. Looking at the molecular orbitals (MOs) responsible for the transition for the As-rich system (Figure 4, Figure S7), it is worth noting that there is little density at the surface where the replaced anions are located for either the HOMO or the LUMO, which is a possible explanation for why the observed redshift is minimal. In order to examine if additional localization can be achieved by the interfacial anion layer at the core/shell interface, the anion-modified models were shelled with ZnSe.

Upon the addition of $\mathrm{ZnSe}$ shell to the InP system (going from $\operatorname{In}_{77} \mathrm{P}_{77}$ to $\operatorname{In}_{10} \mathrm{P}_{10} / \mathrm{Zn}_{67} \mathrm{Se}_{67}$ ) the band gap for the system decreases (by $0.12 \mathrm{eV}$ ), as anticipated due to the ability for the wavefunction to diffuse into the shell. The addition of the anions to the core/shell structure results in an observable red shift of the TD-DFT absorption spectrum (Figure 4). This corresponds to a reduction of the band gaps due to the presence of the additional anions observed in all shelled structures (Figure 5, Table S3). Examination of the density of states (DOS) plot for the $\mathrm{In}_{10} \mathrm{P}_{10} / \mathrm{Zn}_{67} \mathrm{Se}_{67}$ structure (Figure 5) shows that the states around the band gap are comprised predominantly of $\mathrm{P}$ in the valence band and In in the conduction bands as expected for InP systems. However, due to the presence of the $\mathrm{ZnSe}$ shell, there are significant contributions by Se in the valence band and $\mathrm{Zn}$ in the conduction band, showing that the wavefunction is able to diffuse into the shell. The addition of As to the core/shell interface gives rise to As contributions in the valence band. These contributions work to reduce the band gap (by $0.22 \mathrm{eV}$ ) from the $\operatorname{In}_{10} \mathrm{P}_{10} / \mathrm{Zn}_{67} \operatorname{Se}_{67}$ structure. This reduction in the band gap is apparent in the computed TD-DFT absorption spectrum as a redshift (by $0.23 \mathrm{eV}$ ) similar to the process noted experimentally. 


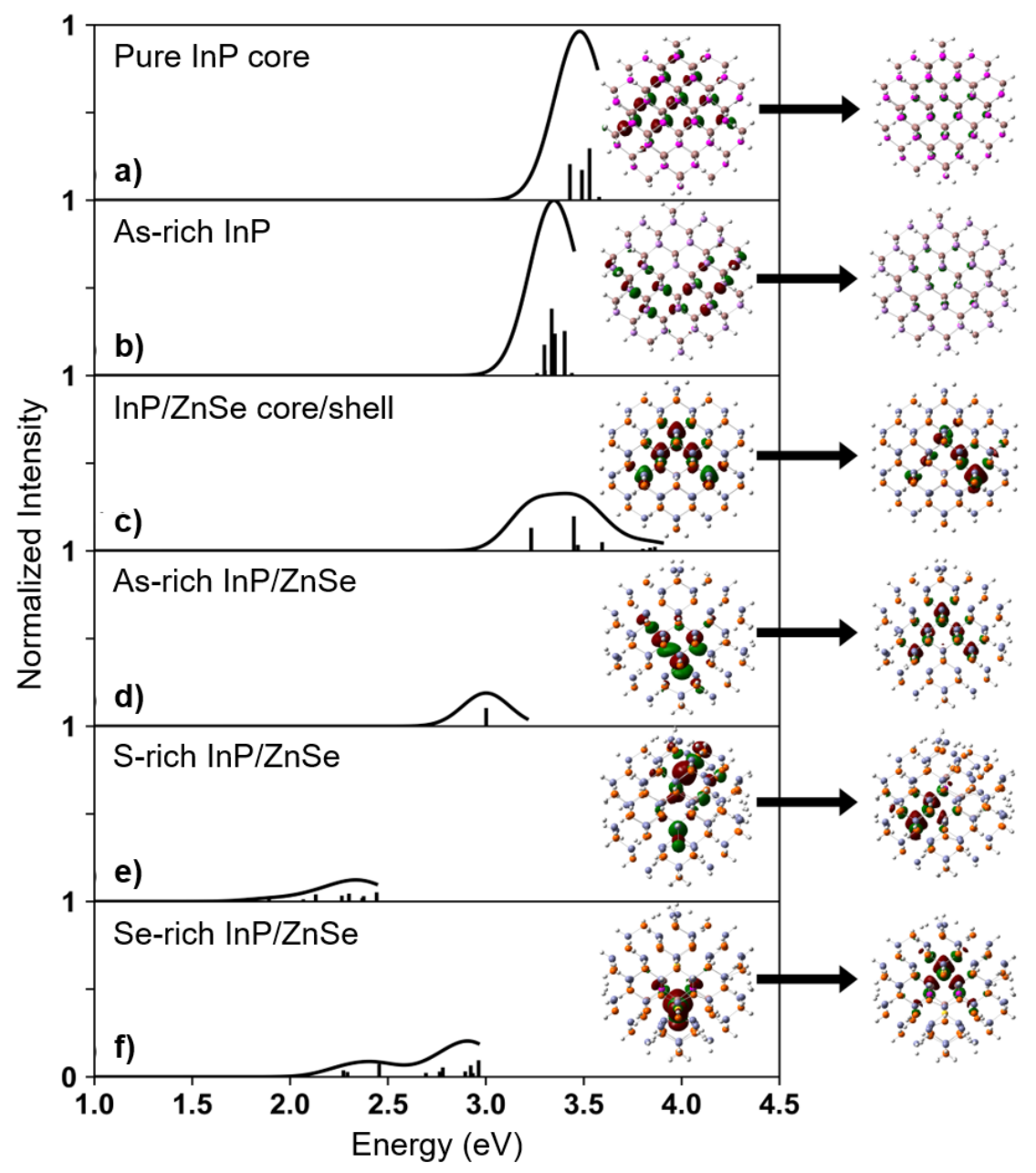

Figure 4. The TD-DFT absorption spectra for (a) the stoichiometric $\operatorname{In}_{77} \mathrm{P}_{77} \mathrm{QD}$, (b) the As-rich $\mathrm{In}_{77} \mathrm{P}_{23} \mathrm{As}_{54} \mathrm{QD},(\mathrm{c})$ the $\mathrm{In}_{10} \mathrm{P}_{10} / \mathrm{Zn}_{67} \mathrm{Se}_{67}$ core/shell QD, (d) the As-rich $\mathrm{In}_{10} \mathrm{P}_{3} \mathrm{As}_{7} / \mathrm{Zn}_{67} \mathrm{Se}_{67} \mathrm{QD}$, (e) the S-rich $\operatorname{In}_{10} \mathrm{P}_{4} \mathrm{~S}_{6} / \mathrm{Zn}_{67} \mathrm{Se}_{67} \mathrm{QD}$, and (f) the Se-rich $\operatorname{In}_{10} \mathrm{P}_{4} \mathrm{Se}_{6} / \mathrm{Zn}_{67} \mathrm{Se}_{67} \mathrm{QD}$. A smoothing function has been applied to the TD-DFT roots (shown as black vertical bars) of $0.12 \mathrm{eV}$, and the resulting spectrum shown as the black line. Atoms are represented as balls with the In atoms in brown, the $\mathrm{P}$ atoms in pink, the $\mathrm{S}$ atoms in yellow, the $\mathrm{Se}$ atoms in orange, the As atoms in purple, the $\mathrm{Zn}$ atoms in gray, and the pseudo $\mathrm{H}$ as white. The leaving and arriving orbitals for the first bright transition ( $\mathrm{f}>0.01$ ) for each system are plotted with an isovalue of 0.02 inset into the spectra. The energetic values for this transition are included in Table S3. 

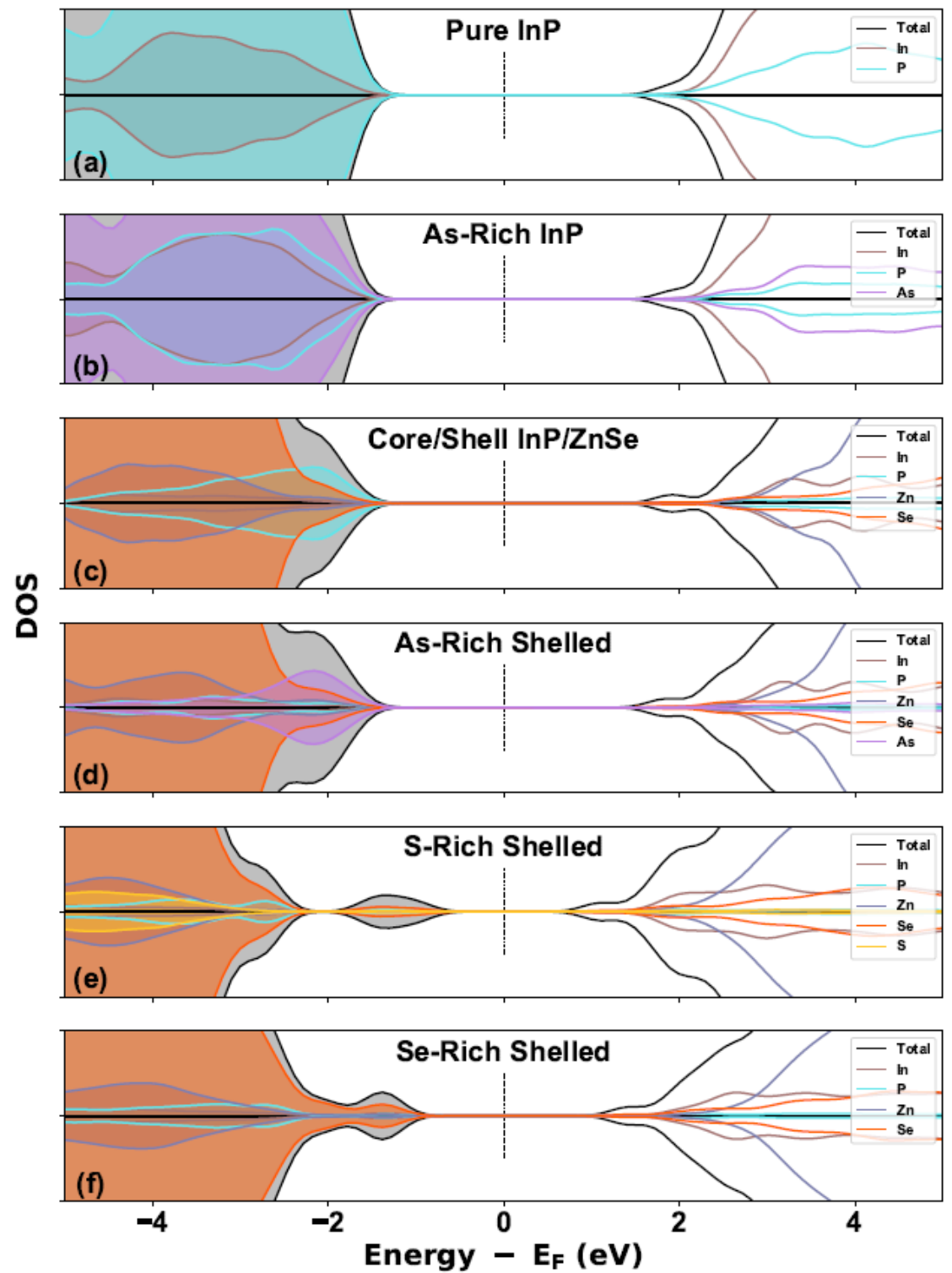

Figure 5. The density of states plots for (a) the stoichiometric $\operatorname{In}_{77} \mathrm{P}_{77} \mathrm{QD}$, (b) the As-rich $\mathrm{In}_{77} \mathrm{P}_{23} \mathrm{As}_{54} \mathrm{QD}$, (c) the $\mathrm{In}_{10} \mathrm{P}_{10} / \mathrm{Zn}_{67} \mathrm{Se}_{67}$ core/shell QD, (d) the As-rich $\mathrm{In}_{10} \mathrm{P}_{3} \mathrm{As}_{7} / \mathrm{Zn}_{67} \mathrm{Se}_{67} \mathrm{QD}$, (e) the S-rich $\operatorname{In}_{10} \mathrm{P}_{4} \mathrm{~S}_{6} / \mathrm{Zn}_{67} \mathrm{Se}_{67} \mathrm{QD}$, and (f) the Se-rich $\mathrm{In}_{10} \mathrm{P}_{4} \mathrm{Se}_{6} / \mathrm{Zn}_{67} \mathrm{Se}_{67} \mathrm{QD}$. The atomic P-orbital contributions by each atomic species is noted in color as contributing to the total density of states (in black). The DOS plots have had a gaussian smoothing of $0.2 \mathrm{eV}$ applied. Positive and negative values correspond to spin up and down, respectively. 
The addition of Se into the interface between the core and shell reduces the band gap even further (by $1.02 \mathrm{eV}$ ) from the $\operatorname{In}_{10} \mathrm{P}_{10} / \mathrm{Zn}_{67} \mathrm{Se}_{67}$ structure. As shown in Figure 5, the Se contributes to states that begin to separate from the edge of the valence band and begin to form shallow holetrap states resulting in the TD-DFT absorption spectrum being significantly redshifted (by 0.96 $\mathrm{eV}$ ) from the $\mathrm{In}_{10} \mathrm{P}_{10} / \mathrm{Zn}_{67} \mathrm{Se}_{67}$ structure. The addition of $\mathrm{S}$ in the core/shell interface leads to a similar result, though the shifts are more pronounced (band gap is redshifted by $1.62 \mathrm{eV}$, absorption is red-shifted by $1.34 \mathrm{eV}$ ) as the states introduced to the band edge are further separated from the conduction band. It is important to note that similar band-edge states have been noted to form in computational InP cluster models before. ${ }^{47}$ In this case, however, these states arose due to the surfaces of the InP models giving rise to spin densities localized to surface metal centers leading to high-spin ground states. To ensure this was not occurring, the energies of several (singlet, $\mathrm{S}=0$, through nonet, $\mathrm{S}=9 / 2$ ) were computed (Figure S8), and it was found that the singlet configuration is the most stable. This is expected for these systems and indicates that there is a different mechanism for the appearance of these states.

Localization of the band-edge MOs to the anions was observed in the core/shell structures (Figure S7). This localization introduces unique states in the doped systems that are dependent on the atomic composition of the core/shell interface. In the case of the As-rich system, these states are still within the conduction band, however for the Se- and S-rich systems these introduced states are above the conduction band resulting in a significant red-shift of the absorption spectrum, and thus the appearance of hole-trap states that have been experimentally noted.

\section{Conclusion and Future Work.}


To summarize, we have synthesized a series of InP and InP/ZnSe QDs with anion-rich interfaces using reactive trimethylsilyl reagents and confirmed successful modification using NMR spectroscopy and elemental analysis. We have correlated these surface chemistry modifications with the resulting steady state and time-resolved PL properties of the QDs. UV-Vis and steady-state PL indicate that anion treatment of the InP cores induces delocalization of the exciton wavefunction and relaxation of core confinement. InP/ZnSe QDs prepared with modulated interfaces result in similar PL decay dynamics on the ns timescale, while the relatively low in PL QYs suggest the creation of new hole traps or non-radiative recombination channels. Although the standardized ZnSe shelling procedure did not result in improved PL properties for anion-doped InP QDs, we have successfully demonstrated surface-limited reaction chemistry with trimethylsilyl anion reagents as a method to deposit submonolayer inorganic shells and obtain a degree of control over shell growth and final absorption and PL profiles. These results introduce a useful method for interfacial control and show that the dynamics are not dramatically impacted at the ns timescale; but PL QYs are still highly sensitive to interfacial composition. DFT calculations show that shallow hole traps are introduced that depend on the atomic composition of the core/shell interface. With increasing complexity and high applicability of QD materials, this work opens doors to atomistic control of the surface and interfaces of nanoscale materials.

\section{Acknowledgements}

This material is based upon work supported by the National Science Foundation under Grant No. MPS-1936100 (NP and BC) and DMR-1719797 (FWE, MM, RB, AJD, and XL). This research was supported by the U.S. National Science Foundation (NSF) through the UW Molecular Engineering Materials Center (MEM-C), a Materials Research Science and Engineering Center 
(DMR-1719797). Part of this work was conducted at the Molecular Analysis Facility, a National Nanotechnology Coordinated Infrastructure site at the University of Washington which is supported in part by the National Science Foundation (Grant No. NNCI-1542101), the University of Washington, the Molecular Engineering and Sciences Institute, and the Clean Energy Institute. The computational work was facilitated though the use of advanced computational, storage, and networking infrastructure provided by the Hyak supercomputer system and funded by the STF at the University of Washington. Computational characterizations of nanomaterials are supported by the National Science Foundation (CHE-1856210 to XL). We gratefully acknowledge the contributions of Dr. Samantha Young in the University of Washington MAF for collecting XRD during the period of restrictions due to the COVID-19 pandemic.

\section{Data Availability Statement}

The data that supports the findings of this study are available within the article and its supplementary material.

\section{TOC Graphic}
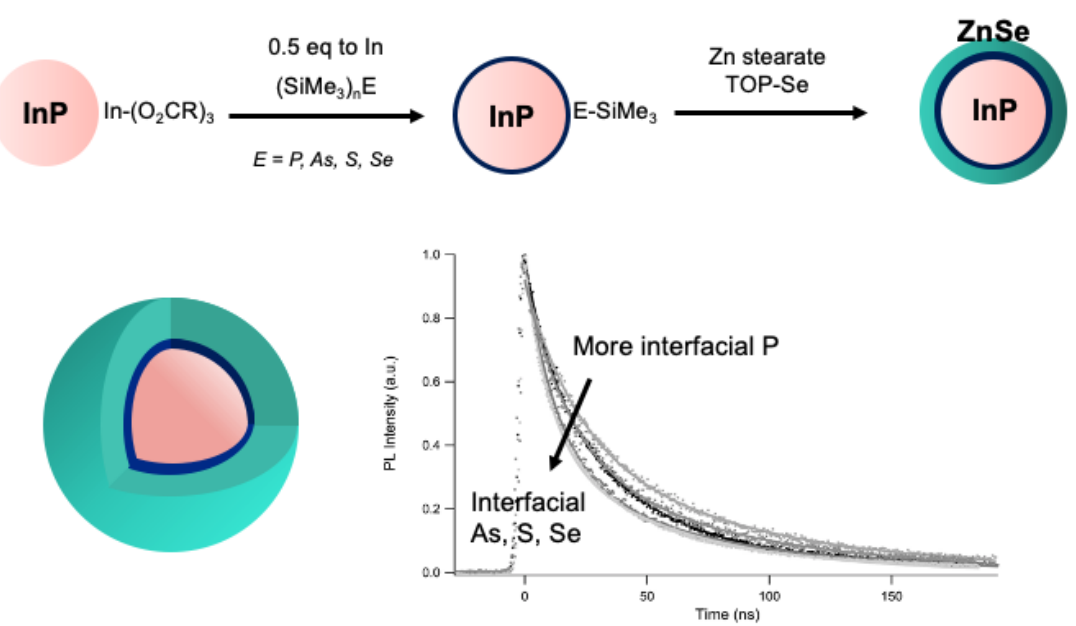


\section{References}

(1) Ippen, C.; Guo, W.; Zehnder, D.; Kim, D.; Manders, J.; Barrera, D.; Newmeyer, B.; Hamilton, D.; Wang, C.; Hotz, C.; Ma, R.; Bin, J.-K.; Kim, B.; Kim, K.; Jang, K.; Park, J.; Lee, T.; Kim, W.-Y.; Lee, J. High Efficiency Heavy Metal Free QD-LEDs for next Generation Displays. Journal of the Society for Information Display 2019, 27 (6), 338346.

(2) Jo, J.-H.; Kim, J.-H.; Lee, K.-H.; Han, C.-Y.; Jang, E.-P.; Do, Y. R.; Yang, H. HighEfficiency Red Electroluminescent Device Based on Multishelled InP Quantum Dots. Opt. Lett. 2016, 41 (17), 3984.

(3) Lim, J.; Park, M.; Bae, W. K.; Lee, D.; Lee, S.; Lee, C.; Char, K. Highly Efficient Cadmium-Free Quantum Dot Light-Emitting Diodes Enabled by the Direct Formation of Excitons within InP@ZnSeS Quantum Dots. ACS Nano 2013, 7 (10), 9019-9026.

(4) Kim, Y.; Ham, S.; Jang, H.; Min, J. H.; Chung, H.; Lee, J.; Kim, D.; Jang, E. Bright and Uniform Green Light Emitting InP/ZnSe/ZnS Quantum Dots for Wide Color Gamut Displays. ACS Appl. Nano Mater. 2019, 2 (3), 1496-1504.

(5) Zhang, H.; Ma, X.; Lin, Q.; Zeng, Z.; Wang, H.; Li, L. S.; Shen, H.; Jia, Y.; Du, Z. HighBrightness Blue InP Quantum Dot-Based Electroluminescent Devices: The Role of Shell Thickness. J. Phys. Chem. Lett. 2020, 11 (3), 960-967.

(6) Lee, T.; Hahm, D.; Kim, K.; Bae, W. K.; Lee, C.; Kwak, J. Highly Efficient and Bright Inverted Top-Emitting InP Quantum Dot Light-Emitting Diodes Introducing a HoleSuppressing Interlayer. Small 2019, 15 (50), 1905162.

(7) Won, Y.-H.; Cho, O.; Kim, T.; Chung, D.-Y.; Kim, T.; Chung, H.; Jang, H.; Lee, J.; Kim, D.; Jang, E. Highly Efficient and Stable InP/ZnSe/ZnS Quantum Dot Light-Emitting Diodes. Nature 2019, 575 (7784), 634-638.

(8) Li, Y.; Hou, X.; Dai, X.; Yao, Z.; Lv, L.; Jin, Y.; Peng, X. Stoichiometry-Controlled InPBased Quantum Dots: Synthesis, Photoluminescence, and Electroluminescence. J. Am. Chem. Soc. 2019, 141 (16), 6448-6452.

(9) Fu, H.; Zunger, A. InP Quantum Dots: Electronic Structure, Surface Effects, and the Redshifted Emission. Physical Review B 1997, 56 (3), 1496.

(10) Adam, S.; Talapin, D. V.; Borchert, H.; Lobo, A.; McGinley, C.; de Castro, A. R. B.; Haase, M.; Weller, H.; Möller, T. The Effect of Nanocrystal Surface Structure on the Luminescence Properties: Photoemission Study of HF-Etched InP Nanocrystals. J. Chem. Phys. 2005, 123 (8), 084706.

(11) Kilina, S. V.; Tamukong, P. K.; Kilin, D. S. Surface Chemistry of Semiconducting Quantum Dots: Theoretical Perspectives. Acc. Chem. Res. 2016, 49 (10), 2127-2135.

(12) Giansante, C.; Infante, I. Surface Traps in Colloidal Quantum Dots: A Combined Experimental and Theoretical Perspective. J. Phys. Chem. Lett. 2017, 8 (20), 5209-5215.

(13) Houtepen, A. J.; Hens, Z.; Owen, J. S.; Infante, I. On the Origin of Surface Traps in Colloidal II-VI Semiconductor Nanocrystals. Chem. Mater. 2017, 29 (2), 752-761.

(14) Cho, E.; Kim, T.; Choi, S.; Jang, H.; Min, K.; Jang, E. Optical Characteristics of the Surface Defects in InP Colloidal Quantum Dots for Highly Efficient Light-Emitting Applications. ACS Appl. Nano Mater. 2018, 1 (12), 7106-7114.

(15) Kovalenko, M. V.; Scheele, M.; Talapin, D. V. Colloidal Nanocrystals with Molecular Metal Chalcogenide Surface Ligands. Science 2009, 324 (5933), 1417-1420. 
(16) Frederick, M. T.; Weiss, E. A. Relaxation of Exciton Confinement in CdSe Quantum Dots by Modification with a Conjugated Dithiocarbamate Ligand. ACS Nano 2010, 4 (6), 3195-3200.

(17) Cossairt, B. M.; Juhas, P.; Billinge, S. J. L.; Owen, J. S. Tuning the Surface Structure and Optical Properties of CdSe Clusters Using Coordination Chemistry. J. Phys. Chem. Lett. 2011, 2 (24), 3075-3080.

(18) Jin, S.; Harris, R. D.; Lau, B.; Aruda, K. O.; Amin, V. A.; Weiss, E. A. Enhanced Rate of Radiative Decay in CdSe Quantum Dots upon Adsorption of an Exciton-Delocalizing Ligand. Nano Lett. 2014, 14 (9), 5323-5328.

(19) Kim, J.-Y.; Kotov, N. A. Charge Transport Dilemma of Solution-Processed Nanomaterials. Chem. Mater. 2014, 26 (1), 134-152.

(20) Boles, M. A.; Ling, D.; Hyeon, T.; Talapin, D. V. The Surface Science of Nanocrystals. Nature Materials 2016, 15 (2), 141-153.

(21) Stein, J. L.; Mader, E. A.; Cossairt, B. M. Luminescent InP Quantum Dots with Tunable Emission by Post-Synthetic Modification with Lewis Acids. J. Phys. Chem. Lett. 2016, 7 (7), 1315-1320.

(22) Kim, T.-G.; Zherebetskyy, D.; Bekenstein, Y.; Oh, M. H.; Wang, L.-W.; Jang, E.; Alivisatos, A. P. Trap Passivation in Indium-Based Quantum Dots through Surface Fluorination: Mechanism and Applications. ACS Nano 2018, 12 (11), 11529-11540.

(23) Hughes, K. E.; Stein, J. L.; Friedfeld, M. R.; Cossairt, B. M.; Gamelin, D. R. Effects of Surface Chemistry on the Photophysics of Colloidal InP Nanocrystals. ACS Nano 2019, 13 (12), 14198-14207.

(24) Toufanian, R.; Piryatinski, A.; Mahler, A. H.; Iyer, R.; Hollingsworth, J. A.; Dennis, A. M. Bandgap Engineering of Indium Phosphide-Based Core/Shell Heterostructures Through Shell Composition and Thickness. Front Chem 2018, 6.

(25) Jang, E.; Kim, Y.; Won, Y.-H.; Jang, H.; Choi, S.-M. Environmentally Friendly InP-Based Quantum Dots for Efficient Wide Color Gamut Displays. ACS Energy Lett. 2020, 5 (4), 1316-1327.

(26) Cao, F.; Wang, S.; Wang, F.; Wu, Q.; Zhao, D.; Yang, X. A Layer-by-Layer Growth Strategy for Large-Size InP/ZnSe/ZnS Core-Shell Quantum Dots Enabling HighEfficiency Light-Emitting Diodes. Chem. Mater. 2018, 30 (21), 8002-8007.

(27) Pietra, F.; De Trizio, L.; Hoekstra, A. W.; Renaud, N.; Prato, M.; Grozema, F. C.; Baesjou, P. J.; Koole, R.; Manna, L.; Houtepen, A. J. Tuning the Lattice Parameter of InxZnyP for Highly Luminescent Lattice-Matched Core/Shell Quantum Dots. ACS Nano 2016, 10 (4), 4754-4762.

(28) Mulder, J. T.; Kirkwood, N.; De Trizio, L.; Li, C.; Bals, S.; Manna, L.; Houtepen, A. J. Developing Lattice Matched ZnMgSe Shells on InZnP Quantum Dots for Phosphor Applications. ACS Appl. Nano Mater. 2020, 3 (4), 3859-3867.

(29) Park, Y.-S.; Bae, W. K.; Padilha, L. A.; Pietryga, J. M.; Klimov, V. I. Effect of the Core/Shell Interface on Auger Recombination Evaluated by Single-Quantum-Dot. Nano Lett. 2014, 14 (2), 396-402.

(30) Freymeyer, N. J.; Click, S. M.; Reid, K. R.; Chisholm, M. F.; Bradsher, C. E.; McBride, J. R.; Rosenthal, S. J. Effect of Indium Alloying on the Charge Carrier Dynamics of ThickShell InP/ZnSe Quantum Dots. J. Chem. Phys. 2020, 152 (16), 161104. 
(31) Rodosthenous, P.; Gómez-Campos, F. M.; Califano, M. Tuning the Radiative Lifetime in InP Colloidal Quantum Dots by Controlling the Surface Stoichiometry. J. Phys. Chem. Lett. 2020, 10124-10130.

(32) Rusishvili, M.; Wippermann, S.; Talapin, D. V.; Galli, G. Stoichiometry of the Core Determines the Electronic Structure of Core-Shell III-V/II-VI Nanoparticles. Chem. Mater. 2020, 32 (22), 9798-9804.

(33) Zhao, Q.; Kulik, H. J. Electronic Structure Origins of Surface-Dependent Growth in III-V Quantum Dots. Chem. Mater. 2018, 30 (20), 7154-7165.

(34) Buckley, J. J.; Couderc, E.; Greaney, M. J.; Munteanu, J.; Riche, C. T.; Bradforth, S. E.; Brutchey, R. L. Chalcogenol Ligand Toolbox for CdSe Nanocrystals and Their Influence on Exciton Relaxation Pathways. ACS Nano 2014, 8 (3), 2512-2521.

(35) Monchen, J. O. V.; Crisp, R. W.; Grimaldi, G.; Bergstein, H. A. C.; du Fossé, I.; van der Stam, W.; Infante, I.; Houtepen, A. J. Finding and Fixing Traps in II-VI and III-V Colloidal Quantum Dots: The Importance of Z-Type Ligand Passivation. J. Am. Chem. Soc. 2018, 140 (46), 15712-15723.

(36) Holder, C. F.; Schaak, R. E. Tutorial on Powder X-Ray Diffraction for Characterizing Nanoscale Materials. ACS Nano 2019, 13 (7), 7359-7365.

(37) Sarkar, A.; Mukherjee, P.; Barat, P. X-Ray Diffraction Studies on Asymmetrically Broadened Peaks of Heavily Deformed Zirconium-Based Alloys. Materials Science and Engineering: A 2008, 485 (1), 176-181.

(38) Snoke, D. W.; Hübner, J.; Rühle, W. W.; Zundel, M. Spin Flip from Dark to Bright States in InP Quantum Dots. Phys. Rev. B 2004, 70 (11), 115329.

(39) Hanrahan, M. P.; Stein, J. L.; Park, N.; Cossairt, B. M.; Rossini, A. J. Elucidating the Location of Cd2+ in Post-Synthetically Treated InP Quantum Dots Using Dynamic Nuclear Polarization 31P and 113Cd Solid-State NMR Spectroscopy. Journal of Physical Chemistry C 2021, 125 (5), 2956-2965.

(40) Vainshtein, I. A.; Zatsepin, A. F.; Kortov, V. S. Applicability of the Empirical Varshni Relation for the Temperature Dependence of the Width of the Band Gap. Phys. Solid State 1999, 41 (6), 905-908.

(41) Lewis, J. E.; Wu, S.; Jiang, X. J. Unconventional Gap State of Trapped Exciton in Lead Sulfide Quantum Dots. Nanotechnology 2010, 21 (45), 455402.

(42) Gaponenko, M. S.; Tolstik, N. A.; Lutich, A. A.; Onushchenko, A. A.; Yumashev, K. V. Temperature-Dependent Photoluminescence Stokes Shift in PbS Quantum Dots. Physica E: Low-dimensional Systems and Nanostructures 2013, 53, 63-65.

(43) M. J. Frisch, G. W. Trucks, H. B. Schlegel, G. E. Scuseria, M. A. Robb, J. R. Cheeseman, G. Scalmani, V. Barone, G. A. Petersson, H. Nakatsuji, X. Li, M. Caricato, A. V. Marenich, J. Bloino, B. G. Janesko, R. Gomperts, B. Mennucci, H. P. Hratchian, J. V. Ortiz, A. F. Izmaylov, J. L. Sonnenberg, D. Williams-Young, F. Ding, F. Lipparini, F. Egidi, J. Goings, B. Peng, A. Petrone, T. Henderson, D. Ranasinghe, V. G. Zakrzewski, J. Gao, N. Rega, G. Zheng, W. Liang, M. Hada, M. Ehara, K. Toyota, R. Fukuda, J. Hasegawa, M. Ishida, T. Nakajima, Y. Honda, O. Kitao, H. Nakai, T. Vreven, K. Throssell, J. A. Montgomery, Jr., J. E. Peralta, F. Ogliaro, M. J. Bearpark, J. J. Heyd, E. N. Brothers, K. N. Kudin, V. N. Staroverov, T. A. Keith, R. Kobayashi, J. Normand, K. Raghavachari, A. P. Rendell, J. C. Burant, S. S. Iyengar, J. Tomasi, M. Cossi, J. M. Millam, M. Klene, C. Adamo, R. Cammi, J. W. Ochterski, R. L. Martin, K. Morokuma, O. 
Farkas,; J. B. Foresman, and D. J. Fox. Gaussian 16, Revision B.01. Gaussian Inc. Wallingford CT 2016.

(44) Perdew, J. P.; Burke, K.; Ernzerhof, M. Generalized Gradient Approximation Made Simple [Phys. Rev. Lett. 77, 3865 (1996)]. Phys. Rev. Lett. 1997, 78 (7), 1396-1396.

(45) Ernzerhof, M.; Scuseria, G. E. Assessment of the Perdew-Burke-Ernzerhof ExchangeCorrelation Functional. J. Chem. Phys. 1999, 110 (11), 5029-5036.

(46) Adamo, C.; Barone, V. Toward Reliable Density Functional Methods without Adjustable Parameters: The PBE0 Model. J. Chem. Phys. 1999, 110 (13), 6158-6170.

(47) Snee, P. T. DFT Calculations of InP Quantum Dots: Model Chemistries, Surface Passivation, and Open-Shell Singlet Ground States. J. Phys. Chem. C 2021, 125 (21), 11765-11772. 